\title{
Study on Optimal Station Distribution Based on TDOA Measurements
}

\author{
Shun Sun ${ }^{1, a}$, Zhiguo Wang ${ }^{2, b}$ and Zhi Wang ${ }^{1, c}$ \\ ${ }^{1}$ Institute of Information Fusion, Naval Aeronautical and Astronautical University, Yantai 264001, \\ China; \\ ${ }^{2}$ School of Electronic Engineering, Beijing University of Posts and Telecommunications, Beijing \\ 100876, China. \\ asunms7@qq.com, bzgwang24@163.com, cwzxxrh@163.com
}

\begin{abstract}
The optimal stations distribution for a single source target is proposed to improve performance of four stations passive location system based on time difference of arrival (TDOA) measurements. Geometrical dilution of precision (GDOP) is obtained by deriving Cramér-Rao Lower Bound (CRLB) firstly. Then three general conclusion are summarized by simulation and analysis about influence of four kinds of typical station distribution on GDOP, and the optimal station distribution is obtained to guide station distribution when multistations excute reconnaissance mission.
\end{abstract}

Keywords: Passive location; TDOA; Station distribution; GDOP.

\section{Introduction}

Passive sources localization is an important research topic in the field of signal processing. It is widely used in radar [1], sonar [2], wireless communication [3], wireless sensor networks[4] and other fields which drew extensive attention of scholars at home and abroad. Location system based on time difference of arrival(TDOA) is a kind of common passive location system, and has the following advantages. (1) Coordinate transformation(such as transformation from Earth-centered Earth-fixed coordinate to North East Down coordinate) can be regarded as translation and rotation of coordinate, and TDOA measurements can be regarded as range difference of arrival(RDOA) measurements through simple conversion. Hence, TDOA location system will not introduce error terms form coordinate transformation not like bearing measurements. (2) Similarly, TDOA location system will not be influenced by attitude error of observers. Therefore, TDOA passive location system has many distinct advantages in practical application, including high reliability, high stability and high precision.

There are several factors affecting the accuracy of TDOA location system. Wang et al. [5] analyzed the influence of measurement error, observers location errors and systematic bias on tri-satellite TDOA location system, which is instructive to design calibration system. Yang et al. [6] discussed the influence of stations distribution and baseline length of four-stations TDOA location system, and describes characteristics of difference stations distribution. But there is no universal conclusions that can be extended to the case of multi stations. Wang et al. [7] analyzed the variety of location precision for the irregular Y-shaped four-stations distribution based on TDOA and drew two conclusions. But the conclusions cannot be extend to other shape of stations distribution as well as the optimal stations distribution shape for single target.

This paper analyzes the influence of typical distribution station shape on TDOA location system performance and draws general conclusions. Then the optimal stations distribution shape for single target is proposed.

\section{Scenario Description}

We consider the scenario in which 4 unmanned aerial vehicle (UAV) observers locate course target using TDOA measurements. When UAVs flying at the same level, state vector of $i$ th UAV 
observer is $\boldsymbol{s}_{i}=\left[\begin{array}{ll}x_{i} & y_{i}\end{array}\right]^{\mathrm{T}}(i=1,2,3,4)$ without considering height, and the unknown emitter source state vector is $\boldsymbol{u}=\left[\begin{array}{ll}x_{0} & y_{0}\end{array}\right]^{\mathrm{T}}$.

The UAVs' safety should be considered when they locate target collaboratively, that is, the distance between the UAVs and the target should not be less than the safe distance. And there should be a continuous and effective communication between the UAVs, that is, the distance between each UAV and its nearest UAV should not be more than the UAV communication range. The above constraints can be expressed as

$$
\begin{aligned}
& d_{i j}=\sqrt{\left(x_{i}-x_{j}\right)^{2}+\left(y_{i}-y_{j}\right)^{2}}, \quad i, j=0,1, \cdots, 4, i \neq j \\
& d_{i 0} \geq R_{m}, \quad i=1,2,3,4 \\
& \min _{i}\left(d_{i j}\right) \leq R_{c}, \quad j=1,2,3,4, i \neq j
\end{aligned}
$$

Where $R_{m}$ is safety distance which refers to the maximum effective range of air to air missiles and set $R_{m}=15 \mathrm{~km}$ in the paper. $R_{c}$ is UAV communication range which is set $4 \mathrm{~km}$.

\section{Geometrical Dilution of Precision}

GDOP is often used to describe the precision of TDOA location system in different parameters conditions. There are mainly two GDOP calculation methods. One is to calculate the total differential measurement equation, then GDOP is obtained by solving the target state error covariance, another is obtaining GDOP by solving the Cramér-Rao Lower Bound (CRLB) based on measurement equation. CRLB represents lower bound of unbiased estimator variance and is expressed specifically as the optimal accuracy of target position estimation in this paper. GDOP is calculated using CRLB, which is more mathematical and statistical.

TDOA measurement is transformed into range differences of arrival (RDOA) measurement. When UAV 1 is regarded as reference observation station, RDOA measurement equation can be obtained as

$r_{i 1}=h_{i 1}\left(s_{0}, s_{1}, s_{i}\right)=d_{i 0}-d_{10}, i=2,3,4$

Considering to the observer location error, the Jacobian of Eq. 4 is

$\nabla h_{i 1}\left(\boldsymbol{s}_{0}, \boldsymbol{s}_{1}, \boldsymbol{s}_{i}\right)=\left[\begin{array}{lll}\boldsymbol{H}_{i 1} & \boldsymbol{G}_{1} & \boldsymbol{G}_{i}\end{array}\right]$

Where

$$
\begin{aligned}
\boldsymbol{H}_{i 1} & =\left[\begin{array}{ll}
\frac{x_{0}-x_{i}}{d_{i 0}}-\frac{x_{0}-x_{1}}{d_{10}} & \frac{y_{0}-y_{i}}{d_{i 0}}-\frac{y_{0}-y_{1}}{d_{10}}
\end{array}\right] \\
\boldsymbol{G}_{1} & =\left[\begin{array}{ll}
\frac{x_{0}-x_{1}}{d_{10}} & \frac{y_{0}-y_{1}}{d_{10}}
\end{array}\right] \\
\boldsymbol{G}_{i} & =\left[\begin{array}{ll}
\frac{x_{i}-x_{0}}{d_{i 0}} & \frac{y_{i}-y_{0}}{d_{i 0}}
\end{array}\right]
\end{aligned}
$$

Measurement sets are defined as

$$
\boldsymbol{Z}=\left[\begin{array}{lll}
z_{21} & z_{31} & z_{41}
\end{array}\right]^{\mathrm{T}}=h(\boldsymbol{s})
$$

Where $\boldsymbol{\psi}=\left[\begin{array}{ll}\boldsymbol{u}^{\mathrm{T}} & \boldsymbol{s}^{\mathrm{T}}\end{array}\right]^{\mathrm{T}}, \boldsymbol{s}=\left[\begin{array}{llll}\boldsymbol{s}_{1}^{\mathrm{T}} & \boldsymbol{s}_{2}^{\mathrm{T}} & \boldsymbol{s}_{3}^{\mathrm{T}} & \boldsymbol{s}_{4}^{\mathrm{T}}\end{array}\right]^{\mathrm{T}}$. And log-likelihood function is

$$
\ln (p(\boldsymbol{Z} \mid \boldsymbol{\psi}))=K-\frac{1}{2}[\boldsymbol{Z}-h(\boldsymbol{\psi})]^{\mathrm{T}} \boldsymbol{R}^{-1}[\boldsymbol{Z}-h(\boldsymbol{\psi})]
$$

$\boldsymbol{R}=c^{2} \nabla^{-1}$

$\nabla_{r r}=\frac{T}{3 \pi} \omega^{3} \frac{\rho^{2}}{1+M \rho}\left(M \boldsymbol{I}_{M-1}-\mathbf{I}_{M-1}\right)$ 
Where $K$ is constant and independent of the $\psi, R$ is the measurement error covariance matrix. Measurement error of every UAV observer is assumed to be zero-mean Gaussian random process. $c$ is the electromagnetic wave propagation velocity. $T$ is the observation period, $\omega$ is the signal bandwidth, $\rho$ is the ratio for the signal to noise, $\boldsymbol{I}_{M-1}$ is the $M-1$ ordered unit matrix and $\boldsymbol{I}_{M-1}$ is the $M-1$ ordered matrix of all ones.

CRLB of the parameter $\psi$ to be estimated [8] is

$$
\begin{aligned}
& \mathrm{CRLB}_{\psi}=-E\left[\frac{\partial^{2} \ln (\boldsymbol{Z} \mid \boldsymbol{\psi})}{\partial \boldsymbol{\psi} \partial \boldsymbol{\psi}^{\mathrm{T}}}\right]^{-1}=\left[\begin{array}{cc}
\boldsymbol{X} & \boldsymbol{Y} \\
\boldsymbol{Y}^{\mathrm{T}} & \boldsymbol{Z}
\end{array}\right] \\
& \boldsymbol{X}=-E\left[\frac{\partial^{2} \ln (\boldsymbol{Z} \mid \boldsymbol{\psi})}{\partial \boldsymbol{u} \partial \boldsymbol{u}^{\mathrm{T}}}\right]=\boldsymbol{H}^{\mathrm{T}} \boldsymbol{R}^{-1} \boldsymbol{H} \\
& \boldsymbol{Y}=-E\left[\frac{\partial^{2} \ln (\boldsymbol{Z} \mid \boldsymbol{\psi})}{\partial \boldsymbol{u} \partial \boldsymbol{s}^{\mathrm{T}}}\right]=\boldsymbol{H}^{\mathrm{T}} \boldsymbol{R}^{-1} \boldsymbol{G} \\
& \boldsymbol{Z}=-E\left[\frac{\partial^{2} \ln (\boldsymbol{Z} \mid \boldsymbol{\psi})}{\partial \boldsymbol{s} \partial \boldsymbol{s}^{\mathrm{T}}}\right]=\boldsymbol{G}^{\mathrm{T}} \boldsymbol{R}^{-1} \boldsymbol{G}+\boldsymbol{R}_{s}^{-1} \\
& \boldsymbol{H}=\left[\begin{array}{lll}
\boldsymbol{H}_{21}^{\mathrm{T}} & \boldsymbol{H}_{31}^{\mathrm{T}} & \boldsymbol{H}_{41}^{\mathrm{T}}
\end{array}\right]^{\mathrm{T}}
\end{aligned}
$$

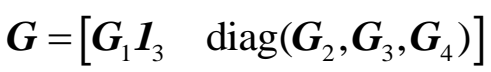

Where diag $(\square)$ represents block diagonal matrix, $\boldsymbol{O}_{2}$ represents second order matrix of zeros, $\boldsymbol{R}_{s}=\sigma_{s}^{2} \boldsymbol{I}_{8}$ is the covariance matrix of observer location error, observer location error is assumed to be the same zero-mean Gaussian distribution, $\boldsymbol{I}_{8}$ is 8 -order unit matrix, $\sigma_{s}^{2}$ is the covariance of observer location error in $x$ or $y$ axis.

Upper-left corner $2 \times 2$ part of $\mathrm{CRLB}_{\psi}$ is related to target location precision, so CRLB of target position $\boldsymbol{u}$ can be obtained as

$$
\mathrm{CRLB}_{\boldsymbol{u}}=\boldsymbol{X}^{-1}+\boldsymbol{X}^{-1} \boldsymbol{Y}\left(\boldsymbol{Z}-\boldsymbol{Y}^{\mathrm{T}} \boldsymbol{X}^{-1} \boldsymbol{Y}\right)^{-1} \boldsymbol{Y}^{\mathrm{T}} \boldsymbol{X}^{-1}
$$

According to the definition of GDOP, then

$$
\mathrm{GDOP}=\sqrt{\sigma_{x_{0}}^{2}+\sigma_{y_{0}}^{2}}=\sqrt{\operatorname{trace}\left(\mathrm{CRLB}_{u}\right)}
$$

Where trace $\left(\mathrm{CRLB}_{u}\right)$ represents the trace of matrix $\mathrm{CRLB}_{u}$.

\section{Analysis}

We shall consider the scenario where four UAVs observers are used to locate an emitting source. Assuming the standard deviation of observers location error is $\sigma_{s}=10 \mathrm{~m}$ for all observers. The total number of samples is $T w / \pi=200000$, where observation period is $T=1$ s. The factor $c / w$ is set to $100 \mathrm{~m}$ and SNR is set to $\rho=1$.Four kinds of typical station distribution shapes, e.g. Square-shaped distribution, Y-shaped distribution, T-shaped distribution and I-shaped distribution, are simulated. And difference station distribution shapes have similar baseline length which is set to UAV communication range $R_{c}=4 \mathrm{~km}$. Simulation scenario is $40 \mathrm{~km} \times 40 \mathrm{~km}$, and simlation results are given in Fig. 1, where the triangular mark indicates observer position and solid circle indicates the optimal target position that meets safety constraint. Details of the observer position, target position and its GDOP are shown in Table 1. 


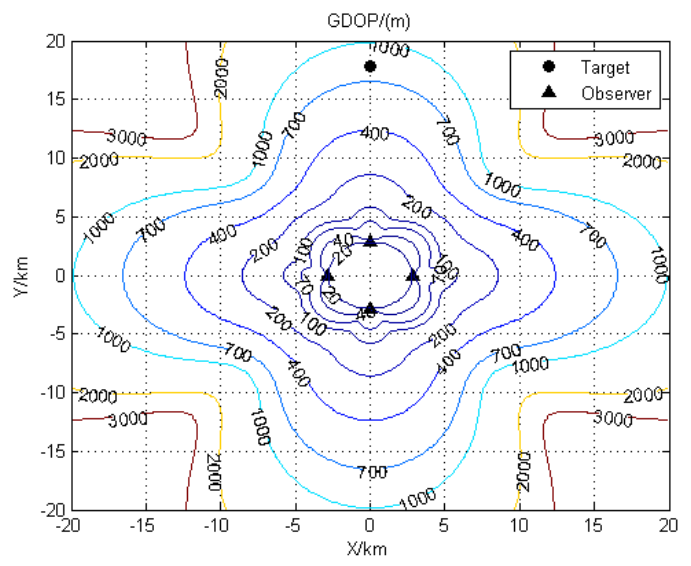

(a) Square-shaped distribution

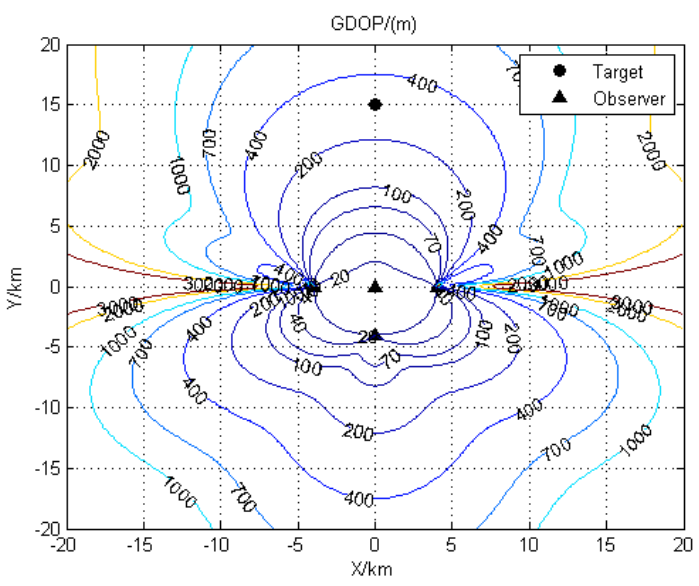

(c) T-shaped distribution

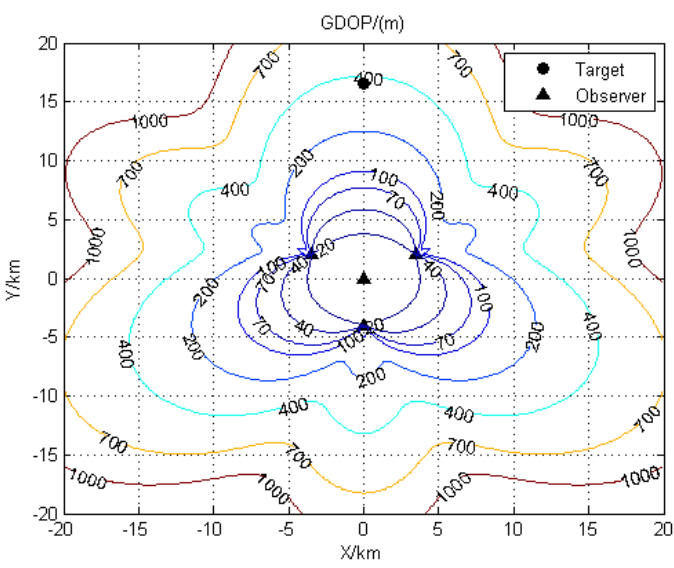

(b) Y-shaped distribution

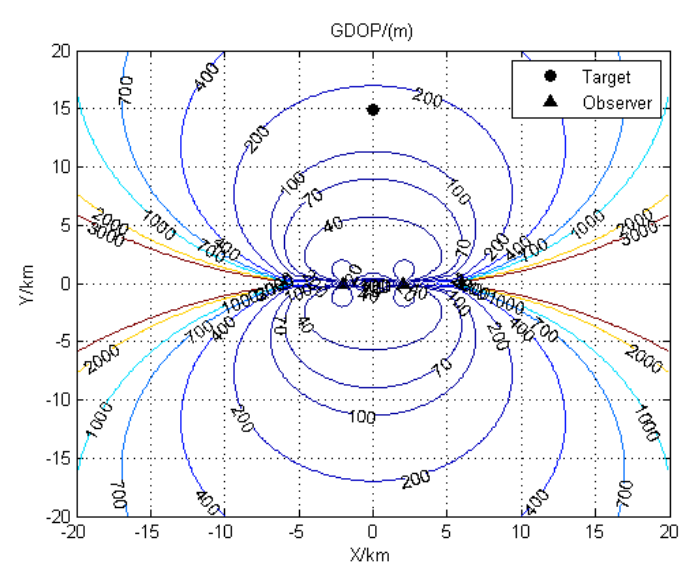

(d) I-shaped distribution

Fig. 1 GDOP diagram using difference station distribution shapes

Table 1 Optimal GDOP of target on difference station distribution

\begin{tabular}{ccccc}
\hline Station distribution & $\begin{array}{c}\text { Square-shaped } \\
\text { distribution }\end{array}$ & $\begin{array}{c}\text { Y-shaped } \\
\text { distribution }\end{array}$ & $\begin{array}{c}\text { T-shaped } \\
\text { distribution }\end{array}$ & $\begin{array}{c}\text { I-shaped } \\
\text { distribution }\end{array}$ \\
\hline Station $1 / \mathrm{km}$ & $(0.00,2.83)$ & $(0.00,0.00)$ & $(0.00,0.00)$ & $(-6.00,0.00)$ \\
Station $2 / \mathrm{km}$ & $(2.83,0.00)$ & $(0.00,-4.00)$ & $(-4.00,0.00)$ & $(-2.00,0.00)$ \\
Station $3 / \mathrm{km}$ & $(0.00,-2.83)$ & $(-3.46,2.00)$ & $(4.00,0.00)$ & $(2.00,0.00)$ \\
Station $4 / \mathrm{km}$ & $(-2.83,0.00)$ & $(3.46,2.00)$ & $(0.00,-4.00)$ & $(6.00,0.00)$ \\
Optimal target position/km & $(0.00,17.83)$ & $(0.00,16.60)$ & $(0.00,15.00)$ & $(0.00,14.87)$ \\
GDOP of target/m & 810.87 & 371.20 & 297.44 & 157.87 \\
\hline
\end{tabular}

In the simulation scenario we set, GDOP of square-shaped distribution and Y-shaped distribution station distribution distributed evenly, particularly GDOP of Y-shaped distribution GDOP is no more than $2000 \mathrm{~m}$. Therefore, when the enemy target position is unknown at the beginning of the reconnaissance, UAVs group can excute reconnaissance mission in accordance with Y-shaped distribution to ensure early discovery and effectively locate source target. T-shaped distribution and I-shaped distribution have high accuracy for targets locating on the y axis, but their GDOP drop significantly and there is obvious ambiguous location for target locating on the $\mathrm{x}$ axis. I-shaped distribution has better location performance on y axis, especially.

Further analysis of the trend of the trough in Fig. 1 shows that distance between the diagonal observers (observer 1 and observer3, observer 2 and observer 4 ) is more farther than other pairs of observers for square-shaped station distribution. And the trough of GDOP diagram is consistent with perpendicular bisectors of the diagonal observers, whose shape is similar to a plus sign. The intersection of two perpendicular bisectors achieves the minimum of GROA. The farthest distance between any pairs of observers is observer 2 and observer 3, observer 3 and observer 4 , and observer 2 and observer 4 for Y-shaped distribution. The relationship between their perpendicular bisectors 
and the trough of GDOP diagram is similar to that for square-shaped distribution. Observer 2 and observer 3 are more farther distance any pairs of observers for T-shaped distribution, and lead to a trough is formed on GDOP diagram. For horizontal I-shaped station distribution, perpendicular bisectors of all pairs of observers parallel to the y axis and distribute on both sides of the $y$ axis symmetrically. Meanwhile, the perpendicular bisector of the two farthest observers coincides with the $y$ axis. This is why I-shaped distribution have the best performance for target location among four typical station distribution shapes.

Through the above simulation and analysis, the following conclusions can be summarized:

Conclusion 1: GDOP in perpendicular bisector of two observers is small, and reaches the minimum at the midpoint of two observers;

Conclusion 2: The farther distance between the two stations, the smaller GDOP in the perpendicular bisector as well as the minimum;

Conclusion 3: GDOP diagram of multi-observers is formed by the superposition of several GDOP diagrams of 2-observers according to the above conclusions. Conclusion 2 indicates that GDOP diagrams of 2-observers whose distance is farther will be dominant component, and corresponding perpendicular bisector forms the trough of GDOP easily.

Based on the above analysis and summary, under the conditions of safety distance constraint of UAVs and communication range constraint, the optimal station distribution for a single target is arc-shaped distribution, that is UAV observation station locating at the circle with target as its center and safety distance as its radius, and the distance between any UAV observers meet the communication range constraint, as shown in Fig. 2 where the dotted line represents the arc with the target as center.

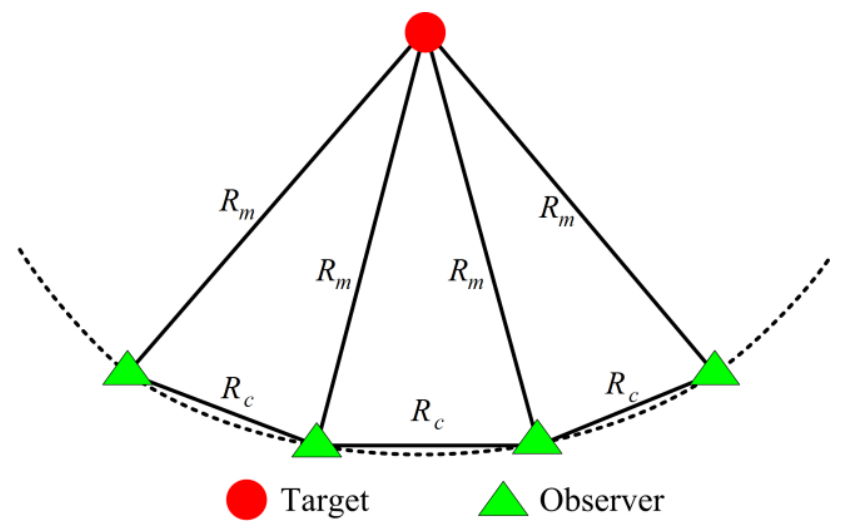

Fig. 2 Optimal station distribution geometry schematic for a single target

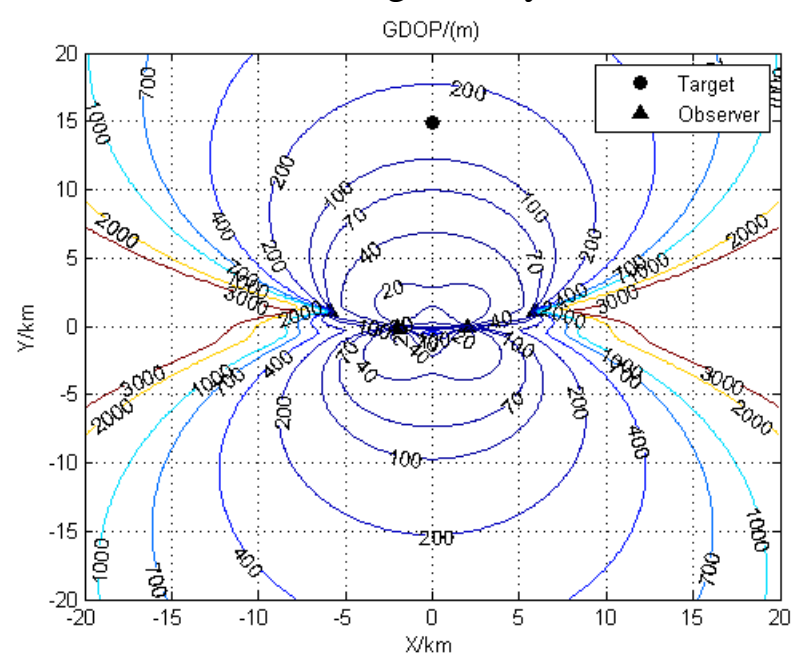

Fig. 3 GDOP diagram using arc-shaped station distribution

When stations distributed as Fig. 2, every station position is the closest to the target under the safety constraint (meet conclusion 1); Distance between any stations is furthest under the communication range constraint (meet conclusion 2); and all perpendicular bisectors of any two 
stations intersect at one point which is target position skillfully (meet conclusion 3). GDOP diagram is simulated according to the simulation conditions of Fig. 1, and results are shown in Fig. 3 and Table 2.

As Fig. 3 shows GDOP diagram using arc-shaped station distribution is similar to I-shaped, but the location accuracy within the arc is higher and that of back area is lower, which meet distribution requirement for a single target. Compared with Table 1 and Table 2, GDOP of optimal target position using arc-shaped station distribution is minimum, only $142.92 \mathrm{~m}$, which further indicate that, the arc-shaped distribution is optimal station distribution for single target based on TDOA location system under the simulation conditions.

Table 2 Optimal GDOP of target for arc-shaped station distribution

\begin{tabular}{cc}
\hline Station distribution & Arc-shaped distribution \\
\hline Station $1 / \mathrm{km}$ & $(-5.86,1.06)$ \\
Station $2 / \mathrm{km}$ & $(-2.00,0.00)$ \\
Station $3 / \mathrm{km}$ & $(2.00,0.00)$ \\
Station $4 / \mathrm{km}$ & $(5.86,1.06)$ \\
Optimal target position $/ \mathrm{km}$ & $(0.00,14.87)$ \\
GDOP of target $/ \mathrm{m}$ & 142.92 \\
\hline
\end{tabular}

\section{Conclusion}

Safe distance constraint and communication range constraint are constructed according to application envieronment. 3 significant conclusions are summarized by simulation and analysis about influence of four kinds of typical four-stations distribution on GDOP. The above conclusions can be generalised to situation of multi-stations distirbution. The optimal station distribution for a single source target is proposed to guide multi-stations reconnaissance mission using efficient station distribution.

\section{References}

[1]. Y. He, J. J. Xiu, J. W. Zhang, et al. Radar data processing with applications, 2nd ed. Publishing House of Electronics Industry, 2009, p. 209-210.

[2]. L. Kumar, R. M. Hegde. Near-field acoustic source localization and beamforming in spherical harmonics domain. IEEE Transactions on Signal Processing. Vol. 64 (2016) No. 13, p. 3351-3361.

[3]. T. S. Rappaport, J. H. Reed, B. D. Woerner. Position location using wireless communications on highways of the future. IEEE Communications Magazine. Vol. 34 (1996) No. 10, p. 33-41.

[4]. P. M. Ghari, R. Shahbazian, S. A. Ghorashi. Wireless sensor network localization in harsh environments using SDP relaxation. IEEE Communications Letters. Vol. 20 (2016) No. 1, p. 137-140.

[5]. Y. G. Wang, Z. L. Chen, T. Li, L. Zheng. Major system error analysis based on tri-satellite TDOA location system. Journal of Spacecraft TT \& C Technology. Vol. 29 (2010) No.1, p. 85-89.

[6]. L. L. Yang, X. W. Sun. Precision analysis of airborne passive location of multi-stations, J. Journal of CAEIT. Vol. 9 (2014) No. 4, p. 348-352.

[7]. H. Wang, D. X. Zhong, Y. Y. Zhou. Location precision analysis in the time difference of arrival location system using irregular distribution. Modern Electronics Technique. Vol. 30 (2007) No. 7, p. $19-21+24$.

[8]. K. C. Ho, X. Lu, L. Kovavisaruch. Source localization using TDOA and FDOA measurements in the presence of receiver location errors: analysis and solution. IEEE Transactions on Signal Processing. Vol. 55 (2007) No. 2, p. 684-696. 